\title{
Achieving adolescent adherence to treatment of major depression
}

This article was published in the following Dove Press journal:

Adolescent Health, Medicine and Therapeutics

31 July 2010

Number of times this article has been viewed

\section{Dennis Staton \\ Lakeland Mental Health Center, Moorhead, MN, USA}

Correspondence: Dennis Staton Lakeland Mental Health Center, 1010 32nd Ave. South, Moorhead, MN 56560, USA

Email dstaton@alum.mit.edu

\begin{abstract}
When treatments are ordered for adolescent major depression, or for other adolescent medical illnesses, adherence and clinical outcomes are likely to be unsatisfactory, unless 4 basic principles of the medical treatment of adolescent illness are implemented. These comprise providing effective patient and parent/caregiver education, establishing effective patient and caregiver therapeutic alliances, providing effective treatment, and managing other factors associated with treatment adherence as indicated. The goals of treatment are to achieve the earliest possible response and remission. Failure to treat adolescent major depression successfully has potentially serious consequences, including worsened adherence, long-term morbidity, and suicide attempt. Accordingly, prescribed treatment must be aggressively managed. Doses of an antidepressant medication should be increased as rapidly as can be tolerated, preferably every 1-2 weeks, until full remission is achieved or such dosing is limited by the emergence of unacceptable adverse effects. A full range of medication treatment options must be employed if necessary. Treatment adherence, occurrence of problematic adverse effects, clinical progress, and safety must be systematically monitored. Adolescents with major depression must be assessed for risk of harm to self or others. When this risk appears significant, likelihood of successful outcomes will be enhanced by use of treatment plans that comprehensively address factors associated with treatment nonadherence. Abbreviated and comprehensive plans for the treatment of potentially fatal adolescent illnesses are outlined in this review.
\end{abstract}

Keywords: adolescent, depression, antidepressant, treatment, adherence, safety

\section{Introduction}

Failure to treat adolescent major depression successfully has potentially serious consequences, including suicide, homicide/suicide (eg, school shooting), severely self-destructive behavior (eg, cutting, burning, allowing sexual victimization and pregnancy), or the development of chronic depression with psychosocial deterioration (eg, school failure, substance abuse, recurrent anger dyscontrol, and dysfunctional interpersonal interactions). A major contributor to treatment failure is nonadherence, ${ }^{1-4}$ which can be enabled by clinician failure to attend to basic principles of the medical treatment of adolescents. The purpose of this review is to describe these principles and to outline treatment strategies that optimize likelihood of achieving satisfactory adherence, obtaining successful outcomes, and insuring safety.

\section{Methods}

Although there is a marked paucity of research about adolescent adherence to treatments for major depression, there are many studies of treatment adherence submit your manuscript | www.dovepress.com

Dovepress

8791 
within other adolescent clinical populations. ${ }^{5}$ These include studies of adolescents with human immunodeficiency virus (HIV) infection, type 1 diabetes, renal failure and transplant, asthma, cystic fibrosis, and inflammatory bowel disease. Data from the latter studies have been included where relevant. Accordingly, the conclusions of this review are intended to be applicable to understanding nonadherence encountered during treatment of any adolescent medical illness. Where information pertaining to adolescents is sparse or unavailable, results of studies of adult clinical populations (often including subjects aged 18 years and older) have been included in order to provide a comprehensive description of issues relevant to treatment nonadherence. The literature referenced in this review was identified using the US National Library of Medicine search engine and is generally limited to the most recently published articles. Older literature is referenced in the included publications. The current status of psychiatric treatment-adherence research is reviewed by Velligan et $\mathrm{al}^{1,2}$ and is not addressed in this paper.

\section{Literature review and discussion Management of adolescent treatment adherence}

Likelihood of achieving long-term remission of adolescent major depression and other adolescent illnesses is significantly enhanced by treatment adherence, ${ }^{1,2,4,6,7}$ assuming correct diagnosis and efficacious treatment. Unfortunately, adolescent compliance with psychiatric and other medication regimens is commonly unsatisfactory. ${ }^{8-11}$ When illness is accompanied by pessimism or hopelessness, achieving adherence may be particularly difficult. How should clinicians manage the probability that adolescent patients will be poorly compliant with treatment recommendations?

Successful management of adolescent treatment adherence has 4 primary components: (1) establishing effective therapeutic alliances, ${ }^{12-19}$ (2) providing effective patient and parent/caregiver education, ${ }^{20,21}$ (3) providing effective treatment, ${ }^{22-25}$ and (4) attending to other relevant factors associated with treatment nonadherence. ${ }^{22,26}$ These 4 components comprise the basic principles of the medical treatment of adolescents and are addressed later, seriatim. Subsequently, the importance of treatment adherence for insuring patient safety is addressed and alternative risk-based treatment protocols are proposed, including one for outpatient management of adolescents who present significant risk of harm to self or others.

\section{Therapeutic alliances}

An unsatisfactory clinician-patient relationship invites treatment nonadherence ${ }^{27-32}$ and adversely impacts treatment outcome. ${ }^{29,30,33}$ Establishing an effective therapeutic alliance requires development of a clinician-patient affective bond, ${ }^{29,34}$ development of patient trust in the clinician's expertise, ${ }^{14,16,19,30,35}$ and collaborative agreement about the treatment plan. ${ }^{29,30,34}$

Establishment of an affective bond is facilitated by clinician warmth, interest, empathy, nonjudgmental acceptance, and honesty, ${ }^{29,30}$ coupled with adolescent patient capacity for interpersonal attachment. ${ }^{36}$ Trust in the clinician's expertise, an important contributor to an effective therapeutic alliance and satisfactory adherence, ${ }^{17-19,21,27,32,37,38}$ is engendered by careful explanatory communication and by early evidence of clinical improvement. Encouraging patient collaboration in treatment planning is important. ${ }^{29,30,39-41}$ Adolescent acceptance of a collaborative role fosters successful coping with concerns about and inconveniences associated with treatment. ${ }^{39}$ Patients with avoidant ${ }^{36}$ or cold/detached ${ }^{42}$ traits will find collaboration difficult and will manifest, at least initially, impaired relationships with clinicians, potentially adversely influencing treatment adherence and outcome.

Therapeutic alliances also must be established with patients' caretakers, ${ }^{29,30}$ recruiting them for compliance support. ${ }^{38,43}$ These alliances promote caretaker cooperation with treatment expectations, ${ }^{29,44}$ more responsible adolescent treatment adherence, ${ }^{29,43}$ and more favorable outcomes. ${ }^{43}$

\section{Patient and parent education}

Adolescents are more likely to comply with treatment if they understand and accept their need to do so. ${ }^{39}$ The goals of patient and caregiver education, therefore, are to generate acceptance of need for treatment, while decreasing apprehension about adverse effects. ${ }^{39}$ Depressed adolescents and their parents should be assumed to be poorly informed about depressive illness and its clinical management, as is the case with many depressed adults. ${ }^{45}$ Individuals with other medical illnesses display widely differing levels of knowledge about their conditions. ${ }^{46}$

Clinicians should explain medical understanding of the illness and its treatment options, including the diagnosis or diagnoses, the known or presumed genesis of the disorder and the acute illness episode, the logic of the recommended treatment, common adverse effects, and the probable consequences of treatment failure vs success. ${ }^{11,21,47-49}$ 
Education should counter misconceptions about the illness, lack of concern about or denial of illness severity, ${ }^{49}$ and misconceptions about the recommended treatment ${ }^{12,23,50-52}$ (especially about the safety of antidepressant medications, discussed later). It also should address other barriers to compliance (eg, concern about cost).

Clinicians should elicit patients' and family members' experiences of problems associated with the illness, the impact of these problems, ${ }^{21}$ and fears regarding the illness and its treatment. It is essential that patients and family members feel listened to and be given hope that the illness is comprehensible and treatable. ${ }^{53}$ Finally, clinicians need to determine how well patient education information has been understood. ${ }^{21}$

Effective patient education also should (1) engender a positive, trusting clinician-patient relationship, ${ }^{14,16,19,35,47}$ (2) engender patient confidence in a decision to accept medication treatment when indicated, ${ }^{48}(3)$ engender a positive attitude about the treatment process and expectation of a successful outcome, ${ }^{15,35,53,54}$ and (4) enhance the likelihood of treatment adherence. ${ }^{15,21,35}$ Providing supportive, empathic clinician-patient dialog, over time, is the most effective means of promoting treatment adherence.

\section{Effective treatment: pharmacotherapy}

This review endorses a medical-model viewpoint that the most rapid, least expensive, and most effective monotherapy for adolescent major depression usually will be antidepressant medication, ${ }^{52,55-58}$ if closely monitored and aggressively managed. ${ }^{56,59,60}$ When there is a family history of mood disorder, including problematic anger dyscontrol, it is particularly sensible for treating clinicians to emphasize the apparent presence of genetic vulnerability for the occurrence of depression. This attributes primary causation to inherited (biologic) bad luck - not to a psychosocial circumstance, or a personality or parenting defect, or to maladaptive depressive thought patterns. It also implies that the condition is medically treatable, even when psychosocial precipitants are clearly present. When patients' symptom and/or family histories suggest underlying pediatric bipolar illness, ${ }^{61,62}$ long-term pharmacotherapy should be anticipated.

\section{Concern about adverse effects}

Expectation of an adverse medication reaction increases the risk of nonadherence, the risk of nonremission of depressive symptoms, and the occurrence of suicidal ideation or attempt. ${ }^{63}$ Expectation of a beneficial response to medication, on the other hand, predicts better treatment adherence. ${ }^{64}$ Consequently, clinicians must effectively confront patient and/or caregiver apprehension about antidepressant medication, which may be reasonable (weight gain and sexual dysfunction) ${ }^{65}$ or unreasonable (altered personality, permanent organ damage, dependence, and greater risk of suicide). ${ }^{12,20,25,64}$

\section{Antidepressant medication and suicidal ideation}

Antidepressant pharmacotherapy ameliorates suicidal ideation $^{55,57,66,67}$ and has a protective effect against completed suicide. ${ }^{68-73}$ Paradoxically, use of antidepressant medication has been reported to have increased the incidence of suicidal thoughts and behaviors by $0.9 \%{ }^{68}$ in child, adolescent, and young adult subjects, in a large database of pharmaceuticalindustry randomized controlled trials (RCTs). ${ }^{51,66,68,71}$ This adverse finding was derived from spontaneously reported (not systematically tracked), retrospectively recovered data. ${ }^{68,71,72,74}$ The limited amount of suicide-related data that was systematically collected during these RCTs revealed no association with antidepressant treatment. ${ }^{71,72}$ Actual suicide attempts were uncommon ${ }^{68}$ and there were no completed child or adolescent suicides in this database. ${ }^{51,68,71}$

No causal explanation for this paradoxical association has been established. ${ }^{68}$ Adolescent reporting of recent suicidal thoughts during RCTs could reflect clinical improvement, ${ }^{74}$ or ascertainment bias, ${ }^{71,72,74}$ or an artifact of sample-selection bias, resulting from the strict inclusion/exclusion criteria of RCTs. ${ }^{75}$ Moreover, conclusions from RCTs cannot be generalized unless they are specifically calibrated to characteristics of target clinical populations. ${ }^{76}$

Antidepressant use is associated with lower suicide rates in adolescents. ${ }^{56,69,72}$ Substantially reduced use of antidepressant medication, since 2004, has been associated with a significant increase in the incidence of child and adolescent completed suicide. ${ }^{71,77}$ In 2 large studies of depressed adolescents not funded by the pharmaceutical industry, antidepressant treatment was not found to increase suicidal ideation or intentional self-injury. ${ }^{57,78}$ Taken altogether, presently available evidence indicates that the benefit of antidepressant use with adolescent major depression is much greater than any potential risk. ${ }^{51,69,71,73,79}$

\section{Systematic monitoring} of pharmacotherapy variables

Medication management requires knowing how patients actually have been taking their medicines, ${ }^{39}$ in addition to determining whether or not progressive clinical improvement 
is taking place, or clinical remission has been achieved, or treatment is failing and there is risk of discontinuation. ${ }^{80}$ The occurrence of unacceptable adverse effects must be promptly detected. ${ }^{12,22,81,82}$ These clinical variables need to be systematically monitored, preferably weekly, until remission of acute illness episodes has been achieved.

The feedback process must be practical and clinically useful for clinicians $;{ }^{80,83}$ it must be sufficiently brief, straightforward, and relevant to insure parent/caretaker completion; ${ }^{83}$ it should enhance patient and caretaker tracking of relevant symptoms $;{ }^{84}$ and it should reflect consensus observations of several informants, where possible. Use of a brief standardized depression rating scale would provide an objective measure of clinical status at each evaluation point. Feedback can be obtained by employing some combination of: (1) frequently scheduled clinician appointments, (2) clinician-office initiated telephone evaluations and adherence support, ${ }^{85,86}$ and (3) strongly encouraged, systematic caretaker reporting.

Assessing adherence in outpatient clinical settings is problematic. Reports regarding adherence often will be inaccurate, even after caretakers have been forewarned that adolescent compliance cannot be trusted and must be closely monitored. Velligan et $\mathrm{al}^{1,2}$ review evaluation options. In selected circumstances, adherence might be monitored by periodic medication plasma concentration determinations, which might or might not encourage adolescent cooperation. Interpretation of results will be difficult, however, because of considerable interindividual pharmacokinetic variability in rates of drug absorption, first-pass metabolism, and subsequent drug elimination. Age, gender, genetic phenotype, stress, and physical activity all influence plasma concentrations and bioavailability. ${ }^{87}$ Clinicians will be unable to distinguish low plasma concentrations caused by poor adherence from low concentrations caused by pharmacokinetic factors, unless investigation by parents/caregivers reveals an adherence problem.

\section{The pharmacotherapy process}

Selection of a selective serotonin reuptake inhibitor (SSRI) other than paroxetine is generally preferred, because of minimal elicitation of side effects, ease of use, greater likelihood of compliance, and safety. ${ }^{38,88-90}$ Once-daily dosing significantly improves medication adherence..$^{22,91,92}$ Medication dosing schedules should be integrated into adolescents' daily routines. Treatment must proceed as rapidly as can be accomplished and must be significantly effective. Inadequate response to an antidepressant agent, or absence of perceived improvement, is a common cause of unsanctioned medication discontinuation. ${ }^{22-25}$ Slow recovery is associated with occurrence of suicidal events. ${ }^{93}$

Major depression is commonly undertreated. ${ }^{94,95}$ In the Treatment for Adolescents with Depression Study (TADS), for example, flexible doses of a single agent (fluoxetine) were slowly increased and limited to a maximum $40 \mathrm{mg} /$ day throughout the first 12 weeks of study. ${ }^{96-98}$ Clinicians were permitted to maintain doses below this maximum in the presence of persisting mild-to-moderate depressive illness. ${ }^{98}$ At 12 weeks, $16.5 \%$ of the TADS fluoxetine-randomized subjects had withdrawn from the study, ${ }^{97}$ residual symptoms were commonly present in the remaining subjects, ${ }^{67,99}$ and $11.9 \%$ of the fluoxetine-randomized subjects had received antidepressant medication outside of the TADS protocol. ${ }^{96}$ At 12 weeks, fluoxetine-condition response and remission rates were only $62 \%$ and $23 \%$, respectively. ${ }^{67,97,99}$ The use of nonaggressive dosing with a single antidepressant agent for 12 weeks clearly reflects suboptimal psychopharmacologic practice.

Although the TADS fluoxetine dose could have been increased to a maximum of $60 \mathrm{mg} /$ day during the succeeding 6 weeks, ${ }^{96}$ at 18 weeks the fluoxetine-condition response and remission rates had risen only to $69 \%$ and $37 \%$, respectively. ${ }^{96,97}$ By 36 weeks, only $50 \%$ of the TADS fluoxetine monotherapy-randomized subjects remained in this treatment arm. ${ }^{97}$ At this evaluation point, the response and remission rates are reported to have risen to $81 \%$ and $55 \%$, respectively, in spite of there having been no TADS-protocol medication adjustments during weeks $19-36 .{ }^{96,97}$ The TADS team attributed these week 36 results to a remarkable delay in medication response, without providing week 12 and week 18 average fluoxetine dose information, or otherwise explaining how insufficient benefit at 12 weeks would have become more impressive over time, in the presence of residual symptoms, high risk for relapse, ${ }^{96,97,99}$ and marked increase in emergency room use and psychiatric hospitalization during weeks $13-36 .^{100}$

The pharmacotherapy protocols of the Adolescent Depression Antidepressant and Psychotherapy Trial (ADAPT) and Treatment of SSRI-Resistant Depression in Adolescents (TORDIA) studies were marred, like that of the TADS trial, by nonaggressive dosing of antidepressant medication: $30 \mathrm{mg} /$ day mean fluoxetine dose at 12 weeks in the ADAPT trial, ${ }^{57}$ and $33.8 \mathrm{mg} /$ day mean dose for fluoxetine and citalopram and $205.4 \mathrm{mg} /$ day mean dose for venlafaxine at 12 weeks in the TORDIA trial, ${ }^{78}$ in spite of 
monotherapy response rates of only $40 \%-45 \%$ after 3 months of treatment. ${ }^{78,101}$

Patients of any age with major depression should be administered doses of antidepressant medicine that are steadily increased until full remission is achieved or such dosing is limited by the emergence of unacceptable adverse effects. Antidepressant dosing should not otherwise be limited, given the severe potential consequences of major depression undertreatment. Doses must be sufficiently high to achieve clinically effective plasma concentrations. Given the marked pharmacokinetic variation that exists among pediatric patients, and the possibility that greater severity of pathology might require higher plasma concentrations, doses sufficient to bring about full remission cannot be predetermined.

Decisions to step up the intensity of treatment should take place every 1-2 weeks, based upon weekly systematic feedback. When further dose increase is unacceptable, but full remission has not been achieved, use of an alternative antidepressant medication, or addition of a second antidepressant to the initially prescribed agent, are potentially effective strategies. When an adolescent has a past history of treatment resistance, combining an antidepressant medication and an augmenting agent, including a second antidepressant, from the initiation of treatment may be advisable. ${ }^{102}$ Thase et al ${ }^{103}$ provide information relevant for clinicians coping with treatment-resistant adolescents.

\section{Management of adverse effects}

Antidepressant adverse effects can include both physiologic symptoms and exacerbation of the symptoms of underlying illnesses (eg, induction of panic symptoms, ${ }^{104}$ or the emergence of manic symptoms or rapid bipolar cycling). The latter may assist clinician identification of comorbid, or underlying, illness. Adverse physiologic symptoms encountered early in treatment tend to become nonproblematic in succeeding weeks; usually these can be managed with education and support. Symptoms of panic exacerbation can be managed with short-term benzodiazepine coverage. Acutely emergent hypomanic or manic symptoms are best treated with an antipsychotic agent; no preferred treatment has been established for rapid bipolar cycling. ${ }^{105}$ An antidepressant medication should not be prematurely discontinued unless this is clearly necessary (eg, following the occurrence of rapid, unmanageable weight gain).

\section{Episodes of nonadherence}

Episodes of treatment nonadherence, when these occur, should be gracefully forgiven and treated as opportunities to examine whether or not the prescribed treatment had been effective - ie, whether or not the subsequent nonadherence has been associated with clinical deterioration. Confirmation of treatment efficacy should be used to augment insight regarding the importance of adherence.

\section{Effective treatment: psychotherapy}

\section{Patient preference for treatment modality}

Major depression is most often treated with individual psychotherapy, ${ }^{106}$ pharmacotherapy, or a combination of both modalities. Cognitive-behavioral therapy (CBT) appears to be more effective for the treatment of depression than other individual psychotherapies, ${ }^{60,107}$ eg, primary care psychosocial support. ${ }^{108}$ Because adolescents tend to attribute their problems to external factors, eg, family dysfunction, ${ }^{29}$ they are likely to prefer psychotherapy. ${ }^{65,109}$

A majority of adults prefer psychotherapeutic treatment for depression, ${ }^{20,94}$ especially when life events or family dysfunction are perceived as causative. ${ }^{20}$ Even when symptoms of depression are perceived as arising from biologic mental illness, adults believe psychotherapy and pharmacotherapy to be comparably appropriate. ${ }^{20}$ Preference for psychotherapy probably reflects predominantly nonmedical understanding of the cause of major depression, ${ }^{20}$ misunderstanding about the mechanism of action of psychiatric medication (eg, treating symptoms but covering underlying problems), ${ }^{20}$ and apprehension about adverse effects of medication, ${ }^{12}$ exacerbated by media reports of alleged problematic outcomes. ${ }^{110}$ Patient and caretaker education will be particularly challenging if adolescents' family members, or social-network peers, have overtly negative attitudes about psychiatric medication.

\section{Psychotherapy}

CBT of adolescent (and adult) depression, when administered by CBT-trained clinicians using research-based protocols, can be effective, ${ }^{111,112}$ although not strongly so. ${ }^{55,97,107}$ Many clinical psychologists and other psychotherapeutic clinicians, however, perhaps the majority, tend not to use optimal, evidence-based interventions. ${ }^{111}$ Considerable evidence indicates that they give more value to their intuition, personal clinical experiences, and informal problem solving. ${ }^{111}$ Thus, depressed adolescents and their families seeking community psychotherapeutic services cannot assume that treatment obtained will be informed by scientific evidence of efficacy. ${ }^{111}$ Moreover, regardless of psychotherapist competence, the probability of beneficial acute response to CBT 
is markedly lowered by the presence of moderately severe or severe major depressive symptoms, economic adversity, and probably also by comorbid conditions. ${ }^{107,113}$

Beneficial response of depression to CBT monotherapy typically occurs 12 or more weeks after initiation of treatment. ${ }^{97}$ At week 12 of the TADS trial, the CBT treatmentcondition results did not differ from those of the placebo condition on any of the 16 TADS endpoint measures ${ }^{113}$ and nearly $60 \%$ of the CBT-treated adolescents were nonresponders. ${ }^{67,113}$ This delay of CBT monotherapy response is unacceptable for moderately or severely depressed adolescents: the earliest achievable response and remission are the goals of treatment. From week 18, or week 24 depending upon TADS endpoint measure, through week 36, the CBT and fluoxetine monotherapy-treatment response rates are reported to have been comparable, ${ }^{97}$ although $29.7 \%$ of the CBT-monotherapy-randomized subjects received antidepressant medication during weeks $13-18$ and $38.7 \%$ received antidepressant medication during weeks 19-36. ${ }^{96}$

There are additional concerns about referral of adolescent major depression for individual psychotherapy. First, to be successful, psychotherapy requires adolescent willingness and capacity to fully cooperate with intensive hour-long, weekly therapy sessions for at least 4 months. This will be problematic with many depressed adolescents..$^{50,114}$ Of 111 adolescents randomized to CBT monotherapy in the TADS trial, only 80 $(72 \%)$ remained in this treatment condition at week 12 and only $55(49.5 \%)$ remained at week $36 .{ }^{97}$ Second, approximately $35 \%-40 \%$ of psychotherapy patients derive no benefit from this treatment and 5\%-10\% deteriorate. ${ }^{80}$ Third, when administered following incidents of self-harm, psychotherapy does not appear to reduce the likelihood of subsequent suicide. ${ }^{115}$

\section{Combination treatment}

The TADS Team strongly endorses combination therapy as the treatment of choice for adolescent major depression. ${ }^{97,116}$ This recommendation is problematic for many reasons: (1) the TADS antidepressant monotherapy treatment was inadequate (as discussed above), (2) adding CBT to pharmacotherapy is unlikely to improve remission rates with adolescents whose major depressions are moderately severe or severe, or associated with low levels of cognitive distortion, ${ }^{117}$ (3) the ADAPT trial found no difference in treatment effectiveness between SSRI monotherapy and SSRI + CBT combination therapy, and no protection from combination treatment against the emergence of suicidal ideation and self-injury ${ }^{57}$ (the TORDIA trial also detected no additional combination-treatment protection against suicidal ideation or self-injury ${ }^{78}$ ), and (4) even when competent, evidence-based individual psychotherapy is available, combination treatment is more expensive than medication monotherapy and likely often to be unaffordable. ${ }^{57}$ The TADS medication plus medication management costs were less than half the average cost of CBT. ${ }^{100}$ The ADAPT trial found combination therapy to be more expensive and no more cost-effective than antidepressant monotherapy, whether or not the hospital costs of 2 combination-treated subjects were included. ${ }^{57}$ Domino et $\mathrm{al}^{100}$ inflated their estimate of total antidepressant monotherapy costs by including disproportionately greater emergency room and psychiatric hospitalization expenses incurred in this treatment arm during weeks 13-36. Had the TADS subjects in this treatment arm been managed more aggressively, including use of a full range of medication treatment options, hospital expenses should have been negligible. Addition of individual psychotherapy to pharmacotherapy may, ${ }^{118-120}$ or may not, ${ }^{57,121}$ be the most effective treatment.

\section{Other predictors of poor treatment adherence}

Successful medical treatment of adolescent illnesses requires management of predictors of poor adherence, particularly those that effective education can modify, and also those related to the presence of comorbid disorders, which can be concurrently treated. Table 1 contains a list of predictors of poor treatment adherence among adolescent (and adult) clinical populations, grouped into categories of related items. Research studies emphasize both predictors of poor treatment compliance and predictors of better, or good, compliance. The latter are included in Table 1 in their opposite form, as predictors of poor adherence.

Many predictors of poor treatment adherence can be ameliorated by effective patient and caretaker education. These predictors are readily identifiable in Table 1 . Other predictors of poor adherence cannot be modified by treating clinicians. Examples of the latter include low level of education ${ }^{81}$ and/or poor English-language proficiency, ${ }^{23}$ psychosocial immaturity, ${ }^{122,123}$ and disadvantaged economic ${ }^{23,82,124}$ and/or family functioning ${ }^{123-125}$ circumstances. Although clinicians always should communicate in a clear, easily understood manner, ${ }^{47,53}$ this is especially necessary in situations where the latter adverse predictors are prominent. Their presence should be borne in mind.

The comorbid presence of attention-deficit hyperactivity disorder (ADHD), and/or significant anxiety, and/or 
Table I Predictors of poor treatment adherence

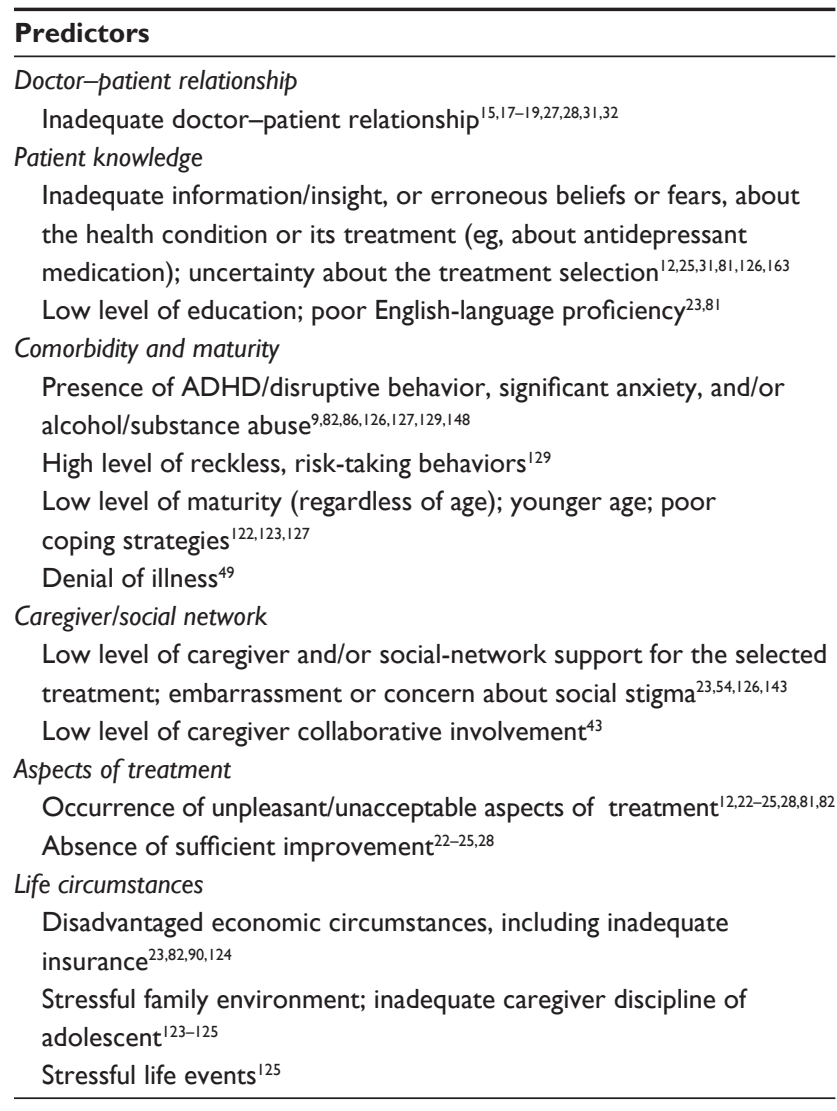

Abbreviation: ADHD, attention-deficit hyperactivity disorder.

substance abuse, in adolescents with major depression, predicts worsened treatment adherence. ${ }^{9,82,86,126,127}$ Prominent symptoms of adolescent ADHD include disorganization and forgetfulness (the latter symptom is reported to be the most common cause of medication nonadherence in adults ${ }^{18,26,128}$ ). Adolescents with ADHD also may exhibit risk-taking behaviors that are associated with poor treatment outcomes. ${ }^{129}$ Significant anxiety in adolescents with major depression may signal the presence of fears of taking medication or attending clinic appointments, which otherwise might not be detected. Comorbidity with ADHD and/or an anxiety disorder is treatable and may need to be concurrently addressed. The presence of substance abuse, especially alcohol abuse, in adolescents with major depression, is an obstacle to effective treatment that requires confrontation and separate therapy. Court-ordered intervention may be necessary. The major depression should be simultaneously treated.

Excessive anger has been identified as a correlate of medication noncompliance during treatment of pediatric illness. ${ }^{130}$ Problematic anger responds to a variety of pharmacologic treatments. ${ }^{131}$
Insuring safety

Assessment for risk of harm to self or others

Adolescents with major depression must be assessed for risk of harm. As many as $15 \%$ of adolescents in the general population experience significant risk of suicide ${ }^{132}$ and approximately 4\% attempt killing themselves. ${ }^{133,134}$ Table 2 contains a combined list of risk factors for adolescent suicide and suicidal homicide. Unfortunately, the presence of many risk factors does not accurately predict danger, nor does their absence insure safety. ${ }^{135}$ Clinical judgments of risk are uncertain.

\section{Relevance of treatment adherence}

Most adolescent suicide attempts are probably impulsive (over $40 \%$ of adult suicide attempts, aged 18 years and older, are reported to be unplanned ${ }^{136}$ ). Because suicide attempts cannot be accurately anticipated, when an evaluation raises concern about the possibility of an attempt, the ensuing treatment plan must emphasize close monitoring of clinical status and compliance with the prescribed treatment. Insufficient treatment response is associated with worsened adherence ${ }^{23,25}$ and suicide attempt ${ }^{137}$ and must be quickly detected. Adherence is critically important because (1) antidepressant discontinuation is associated with significant risk of subsequent suicide attempt $t^{5,138}$ and (2) a majority of treated adolescent suicide victims are reported to have been poorly or noncompliant prior to death. ${ }^{139}$

Those adolescents with a previous history of suicide attempt are at greater risk for subsequent attempts and completed suicide. ${ }^{14-142}$ They typically will have been poorly compliant

Table 2 Predictors of adolescent risk of harm to self or others

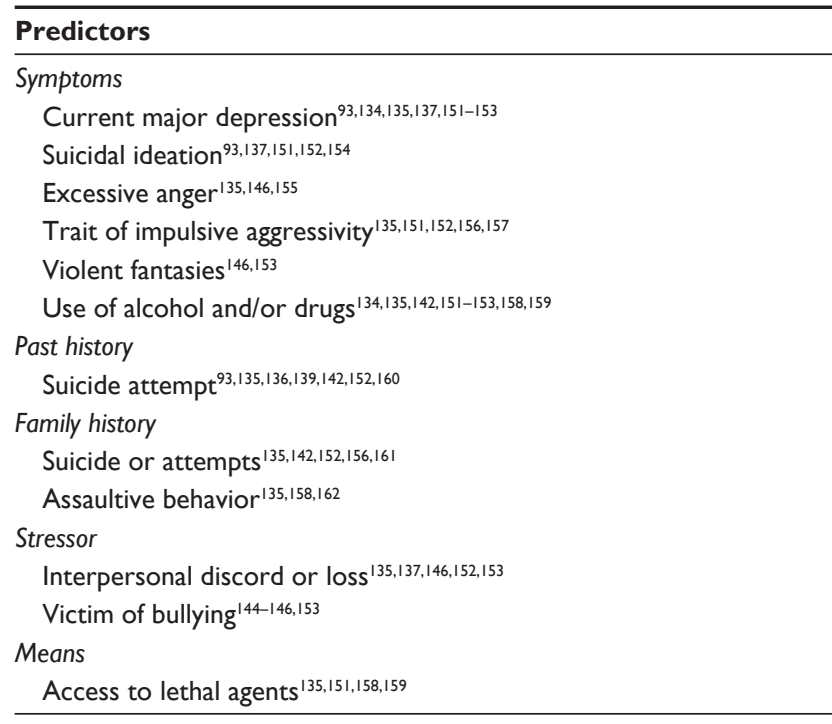


with treatment after past attempts. ${ }^{140}$ When potentially suicidal adolescents also have comorbid ADHD, significant anxiety, and/ or a substance use disorder, they are even less likely to be compliant with treatment. ${ }^{143}$ Adherence and response to treatment must be especially closely monitored with these patients.

\section{Risk of homicide}

A very small percentage of depressed adolescents, typically boys who have been bullied, murder peers or family members. ${ }^{144-146}$ Risk of homicide can arise when there has been bullying by a peer group ${ }^{146}$ or by a family member; and the bullied adolescent is suicidally depressed, expresses vengeful anger, ${ }^{146}$ and has violent fantasies associated with use of media that portrays violent solutions to threatening circumstances. ${ }^{146}$ Angry, depressed adolescent males require close monitoring.

\section{Treatment protocols}

\section{Low risk of suicide or assault}

When risk of harm to self or others appears low, an abbreviated treatment protocol, as outlined in Table 3, should suffice to achieve satisfactory compliance with prescribed treatment. Patient and family education are provided and effective therapeutic alliances are established. Caregivers are recruited for support. Pharmacotherapy is prescribed and adherence, adverse effects, and clinical response are monitored, optimizing medication dose as quickly as feasible, while maintaining awareness that adolescents are likely to be poorly compliant. Even when this appears not to be the case, adherence tends to decrease during a course of treatment ${ }^{147}$ and should be evaluated periodically during long-term maintenance therapy.

\section{Moderate risk of suicide or assault}

When an assessment indicates moderate risk of harm, or other considerations prompt special concern, use of a comprehensive treatment plan is indicated (Table 4). In addition to the components of the abbreviated protocol, comorbid disorders should be identified and simultaneously treated, ${ }^{148}$ if present. Other predictors of poor treatment adherence, listed in Table 1, should be comprehensively evaluated and addressed.22,26,149 Medication management should be accompanied by active psychosocial care, ${ }^{55}$ when indicated.

Depressed adolescents with previous histories of serious suicide attempts should receive comprehensive treatment. Optimizing treatment adherence and efficacy is critically important for these individuals, because community treatment-as-usual does not reduce suicide mortality. ${ }^{141}$

Noncompliance with treatment for serious nonpsychiatric diseases, eg, HIV infection, type I diabetes, or cystic fibrosis, results in morbidity and premature death. The comprehensive strategy (Table 4) is most appropriate for managing the adherence of adolescents who have any potentially fatal pediatric disease.

\section{Apparent high risk of suicide or assault}

When risk of harm appears high, eg, when plans to commit suicide, excessive anger, history of impulsive aggressivity, hopelessness, and substance abuse, are identified, depressed adolescents should be hospitalized, unless caregiver support can insure safety and treatment adherence. Hospitalization will be necessary when a high-risk suicidal adolescent is aggressively hostile towards his caregivers; or is refusing treatment, or appears unlikely to cooperate with close supervision; or if the caregivers appear insufficiently stable, supportive, or responsible to insure safety and treatment adherence. ${ }^{150}$ If safe supervision is assured, the comprehensive treatment protocol outlined in Table 4 should be followed.

\section{Conclusion}

When treatments are ordered for major depression or other serious illnesses in adolescents, adherence to these treatments and

Table 3 Enhancing treatment adherence with adolescents not at risk of suicide or assault

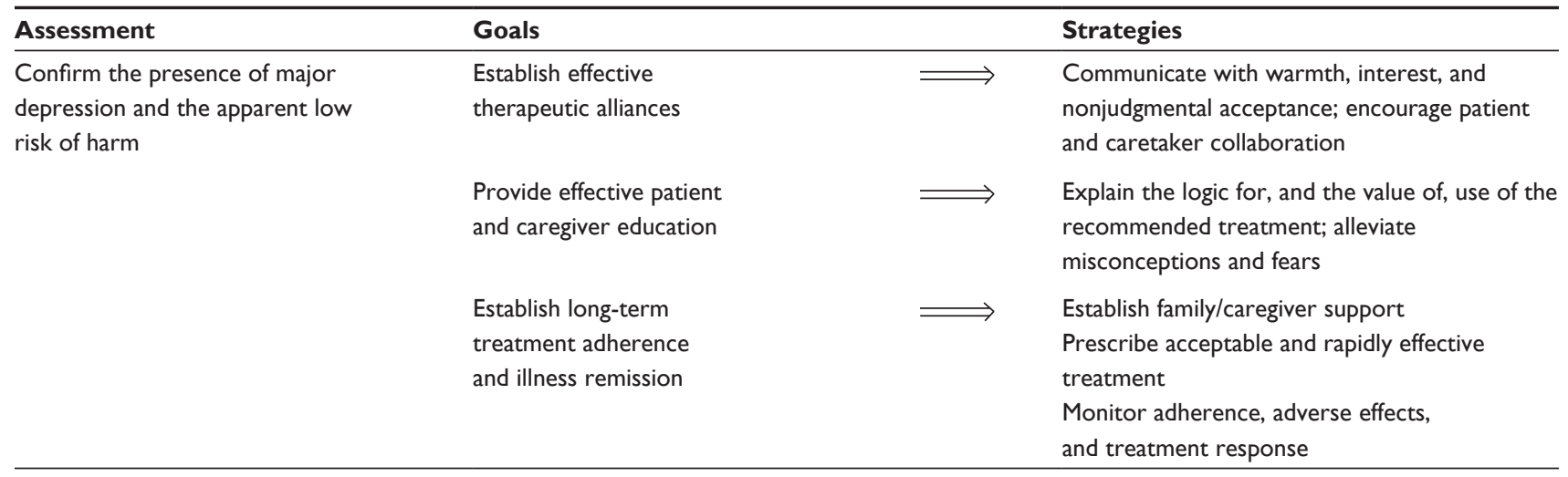


Table 4 Comprehensive strategy for optimizing treatment adherence with potentially suicidal or assaultive adolescents

\begin{tabular}{|c|c|c|}
\hline Assessment & Goals & Strategies \\
\hline $\begin{array}{l}\text { Confirm the presence of major } \\
\text { depression and the apparent } \\
\text { moderate-high risk of harm }\end{array}$ & $\begin{array}{l}\text { Establish effective } \\
\text { therapeutic alliances }\end{array}$ & $\begin{array}{l}\text { Communicate with warmth, interest, and } \\
\text { nonjudgmental acceptance; encourage patient and } \\
\text { caretaker collaboration }\end{array}$ \\
\hline $\begin{array}{l}\text { Identify misconceptions and fears } \\
\text { about the illness or medication }\end{array}$ & $\begin{array}{l}\text { Provide effective patient } \\
\text { and caregiver education }\end{array}$ & $\begin{array}{l}\text { Explain the logic for, and the value of, use of the } \\
\text { recommended treatment; alleviate misconceptions } \\
\text { and fears }\end{array}$ \\
\hline $\begin{array}{l}\text { Identify ADHD, anxiety, substance use } \\
\text { disorders, excessive anger. } \\
\text { impulsive aggressivity }\end{array}$ & $\begin{array}{l}\text { Ameliorate symptoms of } \\
\text { comorbid conditions }\end{array}$ & $\begin{array}{l}\text { Prescribe indicated treatments for comorbid } \\
\text { conditions or problematic symptoms }\end{array}$ \\
\hline $\begin{array}{l}\text { Identify family psychosocial problems } \\
\text { Identify other predictors of poor } \\
\text { treatment adherence }\end{array}$ & $\begin{array}{l}\text { Ensure safety and establish } \\
\text { long-term treatment } \\
\text { adherence and illness } \\
\text { remission }\end{array}$ & $\begin{array}{l}\text { Establish family/caregiver support that ensures } \\
\text { adherence and safety } \\
\text { Prescribe acceptable (including adverse } \\
\text { effects) and rapidly effective treatment } \\
\text { Closely monitor adherence, adverse effects, } \\
\text { treatment response, and safety } \\
\text { Attend to relevant predictors of poor adherence }\end{array}$ \\
\hline
\end{tabular}

Abbreviation: ADHD, attention-deficit hyperactivity disorder.

clinical outcomes are likely to be unsatisfactory unless patient and caregiver education is effective, therapeutic alliances are established, and specific predictors of poor adherence are managed as indicated. When risk of harm to self or others appears significant, likelihood of successful outcomes will be optimized by use of treatment plans that comprehensively address these basic principles of the medical treatment of adolescent illnesses.

The goals of treatment are to achieve the earliest possible response and remission. ${ }^{79}$ Accordingly, treatment must proceed as rapidly and aggressively as can be accomplished and must be significantly effective.

\section{Acknowledgments}

Helpful editorial comments were provided by Bill Duke, $\mathrm{PhD}$, Fargo, ND, and Linda Jo Volness, MSN, Lakeland Mental Health Center, Moorhead, MN.

\section{Disclosure}

The author reports no conflicts of interest in this work.

\section{References}

1. Velligan D, Sajatovic M, Valenstein M, et al. Methodological challenges in psychiatric treatment adherence research. Clin Schizophr Relat Psychoses. 2010;4(1):74-91.

2. Velligan DI, Weiden PJ, Sajatovic M, et al. Assessment of adherence problems in patients with serious and persistent mental illness: recommendations from the Expert Consensus Guidelines. $J$ Psychiatr Pract. 2010;16(1):34-45.

3. Pompili M, Serafini G, Del Casale A, et al. Improving adherence in mood disorders: the struggle against relapse, recurrence and suicide risk. Expert Rev Neurother. 2009;9(7):985-1004.
4. Akerblad AC, Bengtsson F, von Knorring L, Ekselius L. Response, remission and relapse in relation to adherence in primary care treatment of depression: a 2-year outcome study. Int Clin Psychopharmacol. 2006;21(2):117-124.

5. Weiss JJ, Gorman JM. Antidepressant adherence and suicide risk in depressed youth. Am J Psychiatry. 2005;162(9):1756-1757.

6. Hogue A, Henderson CE, Dauber S, Barajas PC, Fried A, Liddle HA. Treatment adherence, competence, and outcome in individual and family therapy for adolescent behavior problems. J Consult Clin Psychol. 2008;76(4):544-555.

7. Akerblad AC, Bengtsson F, Ekselius L, von Knorring L. Effects of an educational compliance enhancement programme and therapeutic drug monitoring on treatment adherence in depressed patients managed by general practitioners. Int Clin Psychopharmacol. 2003;18(6):347-354.

8. Townsend L, Floersch J, Findling RL. Adolescent attitudes toward psychiatric medication: the utility of the Drug Attitude Inventory. J Child Psychol Psychiatry. 2009;50(12):1523-1531.

9. DelBello MP, Hanseman D, Adler CM, Fleck DE, Strakowski SM. Twelve-month outcome of adolescents with bipolar disorder following first hospitalization for a manic or mixed episode. Am J Psychiatry. 2007;164(4):582-590.

10. May DE, Kratochvil CJ, Puumala SE, et al. A manual-based intervention to address clinical crises and retain patients in the Treatment of Adolescents With Depression Study (TADS). JAm Acad Child Adolesc Psychiatry. 2007;46(5):573-581.

11. Staples B, Bravender T. Drug compliance in adolescents: assessing and managing modifiable risk factors. Paediatr Drugs. 2002;4(8):503-513.

12. Chakraborty K, Avasthi A, Kumar S, Grover S. Attitudes and beliefs of patients of first episode depression towards antidepressants and their adherence to treatment. Soc Psychiatry Psychiatr Epidemiol. 2009;44(6):482-488.

13. Dibbelt S, Schaidhammer M, Fleischer C, Greitemann B. Patient-doctor interaction in rehabilitation: the relationship between perceived interaction quality and long-term treatment results. Patient Educ Couns. 2009;76(3):328-335.

14. Ridd M, Shaw A, Lewis G, Salisbury C. The doctor-patient relationship: a synthesis of the qualitative literature on patients' perspectives. $\mathrm{Br} J$ Gen Pract. 2009;59(561):e116-e133.

15. Rubak S, Sandbaek A, Lauritzen T, Borch-Johnsen K, Christensen B. General practitioners trained in motivational interviewing can positively affect the attitide to behaviour change in people with type 2 diabetes. One year follow-up of an RCT, ADDITION Denmark. Scand J Prim Health Care. 2009;27(3):172-179. 
16. Smith SK, Dixon A, Trevena L, Nutbeam D, McCaffery KJ. Exploring patient involvement in healthcare decision making across different education and functional health literacy groups. Soc Sci Med. 2009;69(12):1805-1812.

17. Zolnierek KB, Dimatteo MR. Physician communication and patient adherence to treatment: a meta-analysis. Med Care. 2009;47(8):826-834.

18. D'Inca R, Bertomoro P, Mazzocco K, Vettorato MG, Rumiati R, Sturniolo GC. Risk factors for non-adherence to medication in inflammatory bowel disease patients. Aliment Pharmacol Ther. 2008;27(2):166-172.

19. Green CA, Polen MR, Janoff SL, et al. Understanding how clinicianpatient relationships and relational continuity of care affect recovery from serious mental illness: STARS study results. Psychiatr Rehabil J. 2008;32(1):9-22.

20. Riedel-Heller SG, Matschinger H, Angermeyer MC. Mental disorders - who and what might help? Help-seeking and treatment preferences of the lay public. Soc Psychiatry Psychiat Epidemiol. 2005;40(2):167-174.

21. Maguire P, Pitceathly C. Key communication skills and how to acquire them. BMJ. 2002;325(7366):697-700.

22. Greenley RN, Stephens M, Doughty A, Raboin T, Kugathasan S. Barriers to adherence among adolescents with inflammatory bowel disease. Inflamm Bowel Dis. 2010;16(1):36-41.

23. Hodgkin D, Volpe-Vartanian J, Alegria M. Discontinuation of antidepressant medication among Latinos in the USA. J Behav Health Serv Res. 2007;34(3):329-342.

24. Atkinson MJ, Sinha A, Hass SL, et al. Validation of a general measure of treatment satisfaction, the Treatment Satisfaction Questionnaire for Medication (TSQM), using a national panel study of chronic disease. Health Qual Life Outcomes. 2004;2:12.

25. Haslam C, Brown S, Atkinson S, Haslam R. Patients' experiences of medication for anxiety and depression: effects on working life. Fam Pract. 2004;21(2):204-212.

26. Reynolds NR, Testa MA, Marc LG, et al. Factors influencing medication adherence beliefs and self-efficacy in persons naive to antiretroviral therapy: a multicenter, cross-sectional study. AIDS Behav. 2004;8(2):141-150.

27. Moynihan C, Norman AR, Barbachano Y, et al. Prospective study of factors predicting adherence to medical advice in men with testicular cancer. J Clin Oncol. 2009;27(13):2144-2150.

28. Tamburrino MB, Nagel RW, Chahal MK, Lynch DJ. Antidepressant medication adherence: a study of primary care patients. Prim Care Companion J Clin Psychiatry. 2009;11(5):205-211.

29. Zack SE, Castonguay LG, Boswell JF. Youth working alliance: a core clinical construct in need of empirical maturity. Harv Rev Psychiatry. 2007;15(6):278-288.

30. Green J. Annotation: the therapeutic alliance - a significant but neglected variable in child mental health treatment studies. J Child Psychol Psychiatry. 2006;47(5):425-435.

31. Lopez San Roman A, Bermejo F, Carrera E, Perez-Abad M, Boixeda D. Adherence to treatment in inflammatory bowel disease. Rev Esp Enferm Dig. 2005;97(4):249-257.

32. Sewitch MJ, Abrahamowicz M, Barkun A, et al. Patient nonadherence to medication in inflammatory bowel disease. Am J Gastroenterol. 2003;98(7):1535-1544.

33. Elvins R, Green J. The conceptualization and measurement of therapeutic alliance: an empirical review. Clin Psychol Rev. 2008;28(7):1167-1187.

34. Munder T, Wilmers F, Leonhart R, Linster HW, Barth J. Working Alliance Inventory-Short Revised (WAI-SR): psychometric properties in outpatients and inpatients. Clin Psychol Psychother. 2010;17(3): 231-239.

35. Scott JG, Cohen D, DiCicco-Bloom B, Miller WL, Stange KC, Crabtree BF. Understanding healing relationships in primary care. Ann Fam Med. 2008;6(4):315-322.
36. Morris L, Berry K, Wearden AJ, Jackson N, Dornan T, Davies R. Attachment style and alliance in patients with diabetes and healthcare professionals. Psychol Health Med. 2009;14(5):585-590.

37. Sawada N, Uchida H, Suzuki T, et al. Persistence and compliance to antidepressant treatment in patients with depression: a chart review. BMC Psychiatry. 2009;9:38.

38. Nobile M, Cataldo GM, Marino C, Molteni M. Diagnosis and treatment of dysthymia in children and adolescents. CNS Drugs. 2003;17(13):927-946.

39. Hahn SR. Patient-centered communication to assess and enhance patient adherence to glaucoma medication. Ophthalmology. 2009;116 Suppl 11:S37-S42.

40. Loh A, Leonhart R, Wills CE, Simon D, Harter M. The impact of patient participation on adherence and clinical outcome in primary care of depression. Patient Educ Couns. 2007;65(1):69-78.

41. Schwenk TL, Evans DL, Laden SK, Lewis L. Treatment outcome and physician-patient communication in primary care patients with chronic, recurrent depression. Am J Psychiatry. 2004;161(10): 1892-1901.

42. Hersoug AG, Hoglend P, Havik OE, von der Lippe A, Monsen JT. Pretreatment patient characteristics related to the level and development of working alliance in long-term psychotherapy. Psychother Res. 2009;19(2);172-180.

43. Wysocki T, Nansel TR, Holmbeck GN, et al. Collaborative involvement of primary and secondary caregivers: associations with youths' diabetes outcomes. J Pediatr Psychol. 2009;34(8):869-881.

44. Hawley KM, Weisz JR. Youth versus parent working alliance in usual clinical care: distinctive associations with retention, satisfaction, and treatment outcome. J Clin Child Adolesc Psychol. 2005;34(1):117-128.

45. Johnson S, Dwyer A. Patient perceived barriers to treatment of depression and anxiety in hemodialysis patients. Clin Nephrol. 2008;69(3):201-206.

46. Chapman E, Bilton D. Patients' knowledge of cystic fibrosis: genetic determinism and implications for treatment. $J$ Genet Couns. 2004;13(5):369-385.

47. Shaw A, Ibrahim S, Reid F, Ussher M, Rowlands G. Patients' perspectives of the doctor-patient relationship and information giving across a range of literacy levels. Patient Educ Couns. 2009;75(1):114-120.

48. Stacey D, Menard P, Gaboury I, et al. Decision-making needs of patients with depression: a descriptive study. $J$ Psychiat Ment Health Nurs. 2008;15(4):287-295.

49. Bollini P, Tibaldi G, Testa C, Munizza C. Understanding treatment adherence in affective disorders: a qualitative study. $J$ Psychiatr Ment Health Nurs. 2004;11(6):668-674.

50. Campo JV, Bridge JA. Treatment of youth depression. Am J Psychiatry. 2009;166(9):958-960.

51. Bridge JA, Iyengar S, Salary CB, et al. Clinical response and risk for reported suicidal ideation and suicide attempts in pediatric antidepressant treatment: a meta-analysis of randomized controlled trials. JAMA. 2007;297(15):1683-1696

52. March J, Silva S, Petrycki S, et al. Fluoxetine, cognitive-behavioral therapy, and their combination for adolescents with depression: Treatment for Adolescents with Depression Study (TADS) randomized controlled trial. JAMA. 2004;292(7):807-820.

53. Linder D, Dall'olio E, Gisondi P, et al. Perception of disease and doctor-patient relationship experienced by patients with psoriasis: a questionnaire-based study. Am J Clin Dermatol. 2009;10(5): 325-330.

54. Gonzalez JS, Penedo FJ, Antoni MH, et al. Social support, positive states of mind, and HIV treatment adherence in men and women living with HIV/AIDS. Health Psychol. 2004;23(4):413-418.

55. Goodyer IM, Wilkinson P, Dubicka B, Kelvin R. Forum: the use of selective serotonin reuptake inhibitors in depressed children and adolescents: commentary on the meta-analysis by Hetrick et al. Curr Opin Psychiatry. 2010;23(1):58-61. 
56. Masi G, Liboni F, Brovedani P. Pharmacotherapy of major depressive disorder in adolescents. Expert Opin Pharmacother. 2010;11(3):375-386.

57. Goodyer IM, Dubicka B, Wilkinson P, et al. A randomised controlled trial of cognitive behaviour therapy in adolescents with major depression treated by selective serotonin reuptake inhibitors. The ADAPT trial. Health Technol Assess. 2008;12(14):iii-iv, ix-60.

58. Byford S, Barrett B, Roberts C, et al. Cost-effectiveness of selective serotonin reuptake inhibitors and routine specialist care with and withou cognitive behavioural therapy in adolescents with major depression. Br J Psychiatry. 2007;191(12):521-527.

59. Henke RM, Zaslavsky AM, McGuire TG, Ayanian JZ, Rubenstein LV. Clinical inertia in depression treatment. Med Care. 2009;47(9):959-967.

60. Brent DA. Antidepressants and pediatric depression - the risk of doing nothing. N Engl J Med. 2004;351(16)1598-1601.

61. Staton D, Volness LJ, Beatty WW. Diagnosis and classification of pediatric bipolar disorder. J Affect Disord. 2008;105(1-3):205-212.

62. Staton D. The impairment of pediatric bipolar sleep: hypotheses regarding a core defect and phenotype-specific sleep disturbances. J Affect Disord. 2008;108(3):199-206.

63. Yen CF, Lee Y, Tang TC, Yen JY, Ko CH, Chen CC. Predictive value of self-stigma, insight, and perceived adverse effects of medication for the clinical outcomes in patients with depressive disorders. J Nerv Ment Dis. 2009;197(3):172-177.

64. Stevens J, Wang W, Fan L, Edwards MC, Campo JV, Gardner W. Parental attitudes toward children's use of antidepressants and psychotherapy. J Child Adolesc Psychopharmacol. 2009;19(3):289-296.

65. Bradley KL, McGrath PJ, Brannen CL, Bagnell AL. Adolescents' attitudes and opinions about depression treatment. Commun Ment Health J. 2010;46(3):242-251.

66. Moller HJ, Baldwin DS, Goodwin G, et al. Do SSRIs or antidepressants in general increase suicidality? WPA Section on Pharmacopsychiatry: consensus statement. Eur Arch Psychiatry Clin Neurosci. 2008;258 Suppl 3:3-23.

67. March J, Silva S, Petrycki S, et al. Fluoxetine, cognitive-behavioral therapy, and their combination for adolescents with depression: Treatment for Adolescents with Depression Study (TADS) randomized controlled trial. JAMA. 2004;292(7):807-820.

68. Brent DA. Selective serotonin reuptake inhibitors and suicidality: a guide for the perplexed. Can J Psychiatry. 2009;54(2):72-74.

69. Brent DA. Youth depression and suicide: selective serotonin reuptake inhibitors treat the former and prevent the latter. Can J Psychiatry. 2009;54(2):76-77.

70. Ludwig J, Marcotte DE, Norberg K. Anti-depressants and suicide. $J$ Health Econ. 2009;28(3):659-676.

71. Gibbons RD, Brown CH, Hur K, et al. Early evidence on the effects of regulators' suicidality warnings on SSRI prescriptions and suicide in children and adolescents. Am J Psychiatry. 2007;164(9):1356-1363.

72. Gibbons RD, Hur K, Bhaumik DK, Mann JJ. The relationship between antidepressant prescription rates and rate of early adolescent suicide. Am J Psychiatry. 2006;163(11):1898-1904.

73. Rihmer Z, Akiskal H. Do antidepressants t(h)reat(en) depressives? Toward a clinically judicious formulation of the antidepressantsuicidality FDA advisory in light of declining national suicide statistics from many countries. J Affect Disord. 2006;94(1-3):3-13.

74. Posner K, Oquendo MA, Gould M, Stanley B, Davies M. Columbia Classification Algorithm of Suicide Assessment (C-CASA): classification of suicidal events in the FDA's pediatric suicidal risk analysis of antidepressants. Am J Psychiatry. 2007;164(7):1035-1043.

75. Weisberg HI, Hayden VC, Pontes VP. Selection criteria and generalizability within the counterfactual framework: explaining the paradox of antidepressant-induced suicidality? Clin Trials. 2009;6(2): 109-118.

76. Frangakis $\mathrm{C}$. The calibration of treatment effects from clinical trials to target populations. Clin Trials. 2009;6(2):136-140.
77. Katz LY, Koryrskyj AL, Prior HJ, Enns MW, Cox BJ, Sareen J. Effect of regulatory warnings on antidepressant presciption rates, use of health services and outcomes among children, adolescents and young adults. CMAJ. 2008;178(8):1005-1011.

78. Brent D, Emslie, G, Clarke G, et al. Switching to another SSRI or to venlafaxine with or without cognitive behavioral therapy for adolescents with SSRI-resistant depression: the TORDIA randomized controlled trial. JAMA. 2008;299(8):901-913.

79. March JS. Commentary on 'Forum: use of antidepressants in children and adolescents'. Curr Opin Psychiatry. 2010;23(1):63-65.

80. Lambert MJ, Harmon C, Slade K, Whipple JL, Hawkins EJ. Providing feedback to psychotherapists on their patients' progress: clinical results and practice suggestions. J Clin Psychol. 2005;61(2):165-174.

81. van Geffen EC, van Hulten R, Bouvy ML, Egberts AC, Heerdink ER. Characteristics and reasons associated with nonacceptance of selective serotonin-reuptake inhibitor treatment. Ann Pharmacother. 2008; 42(2):218-225.

82. Akincigil A, Bowblis JR, Levin C, Walkup JT, Jan S, Crystal S. Adherence to antidepressant treatment among privately insured patients diagnosed with depression. Med Care. 2007;45(4)363-369.

83. Kelley SD, Bickman L. Beyond outcomes monitoring: measurement feedback systems in child and adolescent clinical practice. Curr Opin Psychiatry. 2009;22(4):363-368.

84. Feldman-Stewart D, Brundage MD. A conceptual framework for patient-provider communication: a tool in the PRO research tool box. Qual Life Res. 2009;18(1):109-114.

85. Gensichen J, von Korff M, Peitz M, et al. Case management for depression by health care assistants in small primary care practices: a cluster randomized trial. Ann Intern Med. 2009;151(6):369-378.

86. Tay SE. Compliance therapy: an intervention to improve inpatients' attitudes toward treatment. J Psychosoc Nurs Ment Health Serv. 2007;45(6):29-37.

87. Beers MH, Porter RS, Jones TV, Kaplan JL, Berkwits M, editors. The Merck Manual of Diagnosis and Therapy. 18th ed. Whitehouse Station, New Jersey: Merck Research Laboratories; 2006.

88. Mullins CD, Shaya FT, Meng F, Wang J, Bron MS. Comparison of first refill rates among users of sertraline, paroxetine, and citalopram. Clin Ther. 2006;28(2):297-305.

89. Mullins CD, Shaya FT, Meng F, Wang J, Harrison D. Persistence, switching, and discontinuation rates among patients receiving sertraline, paroxetine, and citalopram. Pharmacother. 2005;25(5):660-667.

90. Hansen DG, Vach W, Rosholm JU, Sondergaard J, Gram LF, Kragstrup J. Early discontinuation of antidepressants in general practice: association with patient and prescriber characteristics. Fam Pract. 2004;21(6):623-629.

91. McLaughlin T, Hogue SL, Stang PE. Once-daily bupropion associated with improved patient adherence compared with twice-daily bupropion in treatment of depression. Am J Ther. 2007;14(2):221-225.

92. Stang P, Suppapanaya N, Hogue SL, Park D, Rigney U. Persistence with once-daily versus twice-daily bupropion for the treatment of depression in a large managed-care population. Am J Ther. 2007;14(3):241-246.

93. Brent D, Greenhill LL, Compton S, et al. The Treatment of Adolescent Suicide Attempters Study (TASA): predictors of suicidal events in an open treatment trial. J Am Acad Child Adolesc Psychiatry. 2009. Epub ahead of print.

94. Gonzalez HM, Vega WA, Williams DR, Tarraf W, West BT, Neighbors HW. Depression care in the United States: too little for too few. Arch Gen Psychiatry. 2010;67(1):37-46.

95. Young AS, Klap R, Sherbourne CD, Wells KB. The quality of care for depressive and anxiety disorders in the United States. Arch Gen Psychiatry. 2001;58(1)55-61.

96. Kennard BD, Silva SG, Tonev S, et al. Remission and recovery in the Treatment for Adolescents with Depression Study (TADS) acute and long-term outcomes. J Am Acad Child Adolesc Psychiatry. 2009;48(2):186-195. 
97. The TADS Team. The Treatment for Adolescents with Depression Study (TADS): long-term effectiveness and safety outcomes. Arch Gen Psychiatry. 2007;64(10):1132-1144.

98. The Treatment for Adolescents with Depression Study Team. Treatment for Adolescents with Depression Study (TADS): rationale, design, and methods. J Am Acad Child Adolesc Psychiatry. 2003;42(5):531-542.

99. Kennard B, Silva S, Vitiello B, et al. Remission and residual symptoms after short-term treatment in the Treatment of Adolescents with Depression Study (TADS). J Am Acad Child Adolesc Psychiatry. 2006;45(12):1404-1411.

100. Domino ME, Foster EM, Vitiello B, et al. Relative cost-effectiveness of treatments for adolescent depression: 36-week results from the TADS randomized trial. J Am Acad Child Adolesc Psychiatry. 2009;48(7):711-720.

101. Goodyer I, Dubicka B, Wilkinson P, et al. Selective serotonin reuptake inhibitors (SSRIs) and routine specialist care with and without cognitive behaviour therapy in adolescents with major depression: randomized controlled trial. BMJ. 2007;335(7611):142.

102. Blier P, Ward HE, Tremblay P, Laberge L, Hebert C, Bergeron R. Combination of antidepressant medications from treatment initiation for major depressive disorder: a double-blind randomized study. $\mathrm{Am}$ $J$ Psychiatry. 2010;167(3):281-288.

103. Thase ME, Gaynes BN, Papakostas GI, Trivedi MH, Shelton, RC. Tackling partial remission to depression treatment. J Clin Psychiatry. 2009;70 Suppl 6:4-37.

104. Brauer HR, Nowicki PW, Catalano G, Catalano MC. Panic attacks associated with citalopram. South Med J. 2002;95(9):1088-1089.

105. Amsterdam JD, Wang CH, Shwarz M, Shults J. Venlafaxine versus lithium monotherapy of rapid and non-rapid cycling patients with bipolar II major depressive episode: a randomized, parallel group, open-label trial. J Affect Disord. 2009;112(1-3):219-230.

106. Klomek AB, Stanley B. Psychosocial treatment of depression and suicidality in adolescents. CNS Spectr. 2007;12(2):135-144.

107. Thapar A, Collishaw S, Potter R, Thapar AK. Managing and preventing depression in adolescents. BMJ. 2010;340(c209):254-258.

108. Stein RE, Zitner LE, Jensen PS. Interventions for adolescent depression in primary care. Pediatrics. 2006;118(2):669-682.

109. Dudley AL, Melvin GA, Williams NJ, Tonge BJ, King NJ. Investigation of consumer satisfaction with cognitive-behaviour therapy and sertraline in the treatment of adolescent depression. Aust $N Z J$ Psychiatry. 2005;39(6):500-506.

110. Sajatovic M, Jenkins JH, Cassidy KA, Muzina DJ. Medication treatment perceptions, concerns and expectations among depressed individuals with Type I Bipolar Disorder. J Affect Disord. 2009;115(3):360-366.

111. Baker TB, McFall RM, Shoham V. Current status and future prospects of clinical psychology: toward a scientifically principled approach to mental and behavioral health care. Psychol Sci Public Interest. 2008;9(2):67-103.

112. Klein JB, Jacobs RH, Reinecke MA. Cognitive-behavioral therapy for adolescent depression: a meta-analytic investigation of changes in effect-size estimates. $J$ Am Acad Child Adolesc Psychiatry. 2007;46(11):1403-1413.

113. March J, Silva S, Vitiello B; The TADS Team. The Treatment for Adolescents with Depression Study (TADS): methods and message at 12 weeks. J Am Amer Acad Child Adolesc Psychiatry. 2006;45(12):1393-1403.

114. Jungbluth NJ, Shirk SR. Therapist strategies for building involvement in cognitive-behavioral therapy for adolescent depression. $J$ Consult Clin Psychol. 2009;77(6):1179-1184.

115. Crawford MJ, Thomas O, Khan N, Kulinskaya E. Psychosocial interventions following self-harm: systematic review of their efficacy in preventing suicide. Br J Psychiatry. 2007;190(1):11-17.

116. March JS. Reply to letter to the editor. Arch Gen Psychiatry. 2008;65(6):723-724.
117. Curry J, Rhode P, Simons A, et al. Predictors and moderators of acute outcome in the Treatment for Adolescents with Depression Study (TADS). J Am Acad Child Adolesc Psychiatry. 2006;45(12):1427-1439.

118. Kratochvil CJ, May DE, Silva SG, et al. Treatment response in depressed adolescents with and without co-morbid attentiondeficit/hyperactivity disorder in the Treatment for Adolescents with Depression Study. J Child Adolesc Psychopharmacol. 2009;19(5): 519-527.

119. March JS, Vitiello B. Clinical messages from the Treatment for Adolescents with Depression Study (TADS). Am J Psychiatry. 2009;166(10):1118-1123.

120. Rohde P, Silva SG, Tonev ST, et al. Achievement and maintenance of sustained response during the Treatment for Adolescents with Depression Study continuation and maintenance therapy. Arch Gen Psychiatry. 2008;65(4):447-455.

121. Kocsis JH, Gelenberg AJ, Rothbaum BO, et al. Cognitive behavioral analysis system of psychotherapy and brief supportive psychotherapy for augmentation of antidepressant nonresponse in chronic depression: the REVAMP Trial. Arch Gen Psychiatry. 2009;66(11):1178-1188.

122. Stilley CS, Lawrence K, Bender A, Olshansky E, Webber SA, Dew MA. Maturity and adherence in adolescent and young adult heart recipients. Pediatr Transplant. 2006;10(3):323-330.

123. Mackner LM, Crandall WV. Oral medication adherence in pediatric inflammatory bowel disease. Inflamm Bowel Dis. 2005;11(11):1006-1012.

124. Tan SM, Shafiee Z, Wu LL, Rizal AM, Rey JM. Factors associated with control of type I diabetes in Malaysian adolescents and young adults. Int J Psychiatr Med. 2005;35(2):123-136.

125. Williams PL, Storm D, Montepiedra G, et al. Predictors of adherence to antiretroviral medications in children and adolescents with HIV infection. Pediatrics. 2006;118(6):1745-1757.

126. Neil AL, Batterham P, Christensen H, Bennett K, Griffiths KM. Predictors of adherence by adolescents to a cognitive behavior therapy website in school and community-based settings. J Med Internet Res. 2009;11(1):e6.

127. Murphy DA, Belzer M, Durako SJ, et al. Longitudinal antiretroviral adherence among adolescents infected with human immunodeficiency virus. Arch Pediatr Adolesc Med. 2005;159(8):764-770.

128. Bulloch AG, Adair CE, Patten SB. Forgetfulness: a role in noncompliance with antidepressant treatment. Can J Psychiatry. 2006;51(11):719-722.

129. Bender BG. Risk taking, depression, adherence, and symptom control in adolescents and young adults with asthma. Am J Respir Crit Care Med. 2006;173(9):953-957.

130. Penkower L, Dew MA, Ellis D, Sereika SM, Kitutu JM, Shapiro R. Psychological distress and adherence to the medical regimen among adolescent renal transplant recipients. $\mathrm{Am} \mathrm{J}$ Transplant. 2003;3(11):1418-1425.

131. Mercer D, Douglass AB, Links PS. Meta-analyses of mood stabilizers, antidepressants and antipsychotics in the treatment of borderline personality disorder: effectiveness for depression and anger symptoms. J Pers Disord. 2009;23(2):156-174.

132. Hallfors D, Brodish PH, Khatapoush S, Sanchez V, Cho H, Steckler A. Feasibility of screening adolescents for suicide risk in "real-world" high school settings. Am J Pub Health. 2006;96(2): 282-287.

133. Fortuna LR, Perez DJ, Canino G, Sribney W, Alegria M. Prevalence and correlates of lifetime suicidal ideation and attempts among latino subgroups in the United States. J Clin Psychiatry. 2007;68(4):572-581.

134. Glowinski AL, Bucholz KK, Nelson EC, et al. Suicide attempts in an adolescent female twin sample. J Am Acad Child Adolesc Psychiatry. 2001;40(11):1300-1307.

135. Shain BN; Committee on Adolescence. Suicide and suicide attempts in adolescents. Pediatrics. 2007;120(3):669-676. 
136. Borges G, Angst J, Nock MK, Ruscio AM, Walters EE, Kessler RC. A risk index for 12-month suicide attempts in the National Comorbidity Survey Replication (NCS-R). Psychol Med. 2006;36(12): 1747-1757.

137. Vitiello B, Silva SG, Rohde P, et al. Suicidal events in the Treatment for Adolescents with Depression Study (TADS). J Clin Psychiatry. 2009;70(5):741-747.

138. Valuck RJ, Orton HD, Libby AM. Antidepressant discontinuation and risk of suicide attempt: a retrospective, nested case-control study. J Clin Psychiatry. 2009;70(8):1069-1077.

139. Renaud J, Berlim MT, Seguin M, McGirr A, Tousignant M, Turecki G. Recent and lifetime utilization of health care services by children and adolescent suicide victims: a case-control study. J Affect Disord. 2009;117(3):168-173.

140. Groholt B, Ekeberg O. Prognosis after adolescent suicide attempt: mental health, psychiatric treatment, and suicide attempts in a nine-year follow-up study. Suicide Life Threat Behav. 2009;39(2):125-136.

141. Suominen K, Haukka J, Valtonen HM, Lonnqvist J. Outcome of patients with major depressive disorder after serious suicide attempt. J Cin Psychiatry. 2009;70(10):1372-1378.

142. Kutcher SP, Szumilas M. Youth suicide prevention. Can Med Assoc J. 2008;178(3):282-285.

143. Burns CD, Cortell R, Wagner BM. Treatment compliance in adolescents after attempted suicide: a two-year follow-up study. J Am Acad Child Adolesc Psychiatry. 2008:47(8):948-957.

144. Reuter-Rice K. Male adolescent bullying and the school shooter. J Sch Nurs. 2008;24(6):350-359.

145. Palinkas LA, Prussing E, Landsverk J, Reznik V. Youth-violence prevention in the aftermath of the San Diego East county school shootings: a qualitative assessment of community explanatory models. Ambul Pediatr. 2003;3(5):246-252.

146. Twemlow SW, Fonagy P, Sacco FC, O’Toole ME, Vernberg E. Premeditated mass shootings in schools: threat assessment. J Am Acad Child Adolesc Psychiatry. 2002;41(4):475-477.

147. Gaynor ST, Lawrence PS, Nelson-Gray RO. Measuring homework compliance in cognitive-behavioral therapy for adolescent depression: review, preliminary findings, and implications for theory and practice. Behav Modif. 2006;30(5):647-672.

148. Naar-King S, Idalski A, Ellis D, et al. Gender differences in adherence and metabolic control in urban youth with poorly controlled type I diabetes: the mediating role of mental health symptoms. J Pediatr Psychol. 2006;31(8):793-802.

149. Julius RJ, Novitsky Jr MA, Dubin WR. Medication adherence: a review of the literature and implications for clinical practice. J Psychiatr Pract. 2009; 15(1):34-44.
150. Sorter MT. Adolescents in crisis: when to admit for self-harm or aggressive behavior. Curr Psychiatry. 2010;9(1):35-39,45-46.

151. King CA, O’Mara, RM, Hayward CN, Cunningham RM. Adolescent suicide risk screening in the emergency department. Acad Emerg Med. 2009;16(11):1234-1241.

152. Posner K, Melvin GA, Stanley B, Oquendo MA, Gould M. Factors in the assessment of suicidality in youth. CNS Spectr. 2007;12(2):156-162.

153. Meloy JR, Hempel AG, Mohandie K, Shiva AA, Gray BT. Offender and offense characteristics of a nonrandom sample of adolescent mass murderers. J Am Acad Child Adolesc Psychiatr. 2001;40(6):719-728.

154. King CA, Kerr DC, Passarelli MN, Foster CE, Merchant CR. One-year follow-up of suicidal adolescents: parental history of mental health problems and time to post-hospitalization attempt. $J$ Youth Adolesc. 2010;39(3):219-232.

155. Lee J, Choi H, Kim MJ, Park CG, Shin DS. Anger as a predictor of suicidal ideation in middle-school students in Korea: gender difference in threshold point. Adolescence. 2009;44(174):433-446.

156. Melhem NM, Brent DA, Ziegler M, et al. Familial pathways to earlyonset suicidal behavior: familial and individual antecedents of suicidal behavior. Am J Psychiatry. 2007;164(9):1364-1370.

157. Turecki G. Dissecting the suicide phenotype: the role of impulsiveaggressive behaviours. J Psychiat Neurosci. 2005;30(6):398-408.

158. Zagar RJ, Busch KG, Grove WM, Hughes JR, Arbit J. Looking forward and backward in records for risks among homicidal youth. Psychol Rep. 2009;104(1):103-127.

159. Zagar RJ, Busch KG, Grove WM, Hughes JR. Summary of studies of abused infants and children later homicidal, and homicidal, assaulting later homicidal, and sexual homicidal youth and adults. Psychol Rep. 2009:104(1):17-45.

160. Gehin A, Kabuth B, Pichene C, Vidailhet C. Ten year follow-up study of 65 suicidal adolescents. J Can Acad Child Adolesc Psychiatry. 2009;18(2):117-125.

161. Agerbo E, Nordentoft M, Mortensen PB. Familial, psychiatric, and socioeconomic risk factors for suicide in young people: nested casecontrol study. BMJ. 2002;325(7355):74.

162. Zagar RJ, Isbell SA, Busch KG, Hughes JR. An empirical theory of the development of homicide within individuals. Psychol Rep. 2009;104(1):199-245.

163. Yen $\mathrm{CF}$, Chen $\mathrm{CS}$, Ko $\mathrm{CH}$, et al. Relationships between insight and medication adherence in outpatients with schizophrenia and bipolar disorder: prospective study. Psychiatr Clin Neurosci. 2005;59(4):403-409.

\section{Publish your work in this journal}

Adolescent Health, Medicine and Therapeutics is an international, peer-reviewed, open access journal focusing on health, pathology, and treatment issues specific to the adolescent age group. All aspects of health maintenance, preventative measures and disease treatmen interventions are addressed within the journal and practitioners from

\section{Dovepress}

all disciplines are invited to submit their work as well as healthcare researchers and patient support groups.. The manuscript management system is completely online and includes a very quick and fair peerreview system. Visit http://www.dovepress.com/testimonials.php to read real quotes from published authors. 